\title{
Effects of Rapid or Slow Body Weight Reduction on Intramuscular Protein Degradation Pathways During Equivalent Weight Loss on Rats
}

\author{
Y. NONAKA ${ }^{1}$, S. URASHIMA ${ }^{1}$, M. INAI ${ }^{1}$, S. NISHIMURA ${ }^{1}$, K. HIGASHIDA $^{2}$, S. TERADA $^{1}$ \\ ${ }^{1}$ Department of Life Sciences, Graduate School of Arts and Sciences, The University of Tokyo, \\ Meguro-Ku, Tokyo, Japan, ${ }^{2}$ Department of Food Science and Nutrition, School of Human Cultures, \\ The University of Shiga Prefecture, Hikone-City, Shiga, Japan
}

Received October 10, 2016

Accepted April 7, 2017

On-line July 18, 2017

\section{Summary}

The purpose of this study was to compare the effects of shortterm fasting-induced rapid weight loss with those of slower but equivalent body weight loss induced by daily calorie restriction on muscle protein degradation pathways and muscle protein content. Male Fischer rats were subjected to either $30 \%$ calorie restriction for 2 weeks to slowly decrease body weight (Slow) or 3-day fasting to rapidly decrease body weight by a comparable level of that of the Slow group (Rapid). The final body weights were about $15 \%$ lower in both the Slow and Rapid groups than in the Con group $(p<0.001)$. The total protein content and wet weight of fast-twitch plantaris muscle, but not slow-twitch soleus muscle, were significantly lower in the Rapid group compared with the control rats fed ad libitum. Substantial increases in the expression ratio of autophagosomal membrane proteins (LC3-II/-I ratio) and polyubiquitinated protein concentration, used as biomarkers of autophagy-lysosome and ubiquitinproteasome activities, respectively, were observed in the plantaris muscle of the Rapid group. Moreover, the LC3-II/-I ratio and polyubiquitinated protein concentration were negatively correlated with the total protein content and wet weight of plantaris muscle. These results suggest that short-term fastinginduced rapid body weight loss activates autophagy-lysosome and ubiquitin-proteasome systems more strongly than calorie restriction-induced slower weight reduction, resulting in muscular atrophy in fast-twitch muscle.

\section{Key words}

Skeletal muscle • Fasting • Autophagy-lysosome • Calorie restriction • Ubiquitin-proteasome

\section{Corresponding author}

Y. Nonaka, Department of Life Sciences, Graduate School of Arts and Sciences, The University of Tokyo, 3-8-1 Komaba, Meguro-ku, Tokyo, 153-8902, Japan. Fax: +81-3-5454-4317. E-mail: y-nonaka02@hotmail.co.jp

\section{Introduction}

Many athletes restrict their caloric intake to improve their force-to-mass ratio, to achieve a certain body mass category, or for aesthetic reasons. In particular, athletes in weight-classified sports such as wrestling and boxing usually lose body weight rapidly before competitions (Choma et al. 1998, Reljic et al. 2013). The rapid weight loss, also known as "weight cutting", typically involves several-day fasting until the targeted weight is met. However, fasting is a recognized stimulus of skeletal muscle atrophy (Jagoe et al. 2002), which results in a significant loss of lean body mass that compromises exercise performance. Muscle atrophy occurs when rate of protein degradation exceeds that of protein synthesis. There are two major protein degradation pathways in skeletal muscle. One, the ubiquitin-proteasome pathway, plays a major role in selective protein degradation and serves as the primary 
degradation route for most short-lived proteins (Rock et al. 1994). The other, the autophagy-lysosome pathway, is an intracellular bulk degradation system that is responsible for the degradation of most long-lived proteins, as well as some organelles (Mortimore and Pösö 1987). Both proteolytic pathways become activated during fasting to maintain amino acid pools, leading to muscle atrophy (Mitch and Goldberg 1996, Bujak et al. 2015).

An alternative dietary weight-loss approach practiced by athletes is daily calorie restriction, which results in slower body weight loss compared with fasting. Many Japanese bodybuilders empirically believe that the slower body weight loss induced by daily calorie restriction has less atrophic effects on skeletal muscle than the fasting-induced rapid weight loss and therefore adopt the slower body weight-loss strategy before competitions. However, it remains unclear which type of body weight loss more strongly activates the autophagylysosome and ubiquitin-proteasome pathways and induces muscle atrophy when body weight is reduced to the same extent, because no study has directly compared the effects of rapid vs. slow body weight reduction on the major protein degradation pathways and on protein content in skeletal muscle. Thus, the purpose of this study was to directly compare the effects of rapid or slow body weight loss on the autophagy-lysosome and ubiquitinproteasome pathways and on protein content in rat skeletal muscle during an equivalent weight loss.

\section{Methods}

\section{Animal treatment}

Nineteen-week-old male Fischer-344 rats were obtained from Japan SLC (Shizuoka, Japan) and individually housed under a 12:12-h light:dark cycle (light 09.00-21.00 h) in an air-conditioned room $\left(23^{\circ} \mathrm{C}\right)$. Rats were given a standard laboratory diet ad libitum (CE-2; CLEA Japan, Tokyo, Japan) and water and acclimated to the housing facility for 1 week.

After the acclimation period, the rats were divided into three groups, matched for body weight: one group continued to receive the standard diet ad libitum for the entire 14-day experimental period (Con; $n=5$ ); a second group received the standard diet equal to $70 \%$ of the average amount of food eaten by the Con group during the 14 days to decrease their body weight slowly (Slow; $\mathrm{n}=5$ ); the third group was fed the standard diet ad libitum for 11 days and fasted thereafter for the last
3 days of the study period to rapidly decrease their body weight to a comparable extent as that of the Slow group (Rapid; $n=5$ ). All rats were allowed to drink water freely during the 14-day dietary intervention. Body weight and food intake were recorded daily during the dietary intervention.

At the end of the dietary intervention, fast-twitch plantaris, extensor digitorum longus (EDL), and slowtwitch soleus muscles were quickly and carefully dissected out under anesthesia with isoflurane immediately after the 12-h dark period during which rats eat most food. The muscle samples were weighed, quickly frozen in liquid $\mathrm{N}_{2}$, and stored at $-80{ }^{\circ} \mathrm{C}$ until analysis. After the blood samples were collected from the heart, intra-abdominal fat (sum of the epididymal, mesenteric, and retroperitoneal fat pads) was removed and weighed. The experimental protocols were approved by the Animal Experimental Committee of The University of Tokyo.

\section{Muscle homogenization}

Frozen plantaris and soleus muscles were homogenized in ice-cold Radio-Immuno Precipitation Assay (RIPA) lysis buffer (EMD Millipore, Temecula, CA, USA) containing $50 \mathrm{mM}$ Tris- $\mathrm{HCl}, \mathrm{pH} 7.4,150 \mathrm{mM}$ $\mathrm{NaCl}, 0.25 \%$ deoxycholic acid, $1 \%$ NP-40, $1 \mathrm{mM}$ ethylenediaminetetraacetic acid (EDTA), protease inhibitor cocktail (SIGMA-Aldrich, St. Louis, MO, USA), and phosphatase inhibitors (PhosSTOP; Roche, Basel, Switzerland). The homogenates were frozen and thawed three times to disrupt intracellular organelles and rotated end-over-end at $4{ }^{\circ} \mathrm{C}$ for $60 \mathrm{~min}$ to solubilize the protein. Total protein content per muscle was measured with a bicinchoninic acid (BCA) protein assay kit (Pierce, Rockford, IL, USA). Homogenized samples were then centrifuged at $700 \mathrm{xg}$ for $5 \mathrm{~min}$ at $4{ }^{\circ} \mathrm{C}$ and the supernatants were harvested.

\section{Western blotting}

Protein concentrations of the supernatant harvested as described above were measured with the BCA protein assay kit. Samples were prepared in Laemmli sample buffer (Wako Pure Chemical, Osaka, Japan) and heated for $5 \mathrm{~min}$ in a heating block at $95{ }^{\circ} \mathrm{C}$. Equal amounts of sample protein were subjected to sodium dodecyl sulfate-polyacrylamide gel electrophoresis (SDS-PAGE) (7.5\% or $15 \%$ resolving gels) and then transferred to polyvinylidene difluoride (PVDF) membranes at $200 \mathrm{~mA}$ for $90 \mathrm{~min}$. After transfer, 
membranes were blocked for $1 \mathrm{~h}$ at room temperature in Tris-buffered saline (TBS) with $0.1 \%$ Tween 20 (TBS-T; $20 \mathrm{mM}$ Tris base, $137 \mathrm{mM} \mathrm{NaCl}, \mathrm{pH}$ 7.6) supplemented with $5 \%(\mathrm{w} / \mathrm{v})$ nonfat powdered milk or $5 \%(\mathrm{w} / \mathrm{v})$ bovine serum albumin. Membranes were incubated overnight at $4{ }^{\circ} \mathrm{C}$ with the primary antibody diluted $1: 1,000$ in TBS-T containing $5 \%$ bovine serum albumin. The primary antibodies used were anti-microtubuleassociated protein light chain 3 (LC3) (Medical \& Biological Laboratories, Nagoya, Japan), anti-phosphop70S6K (Cell Signaling Technology, Danvers, MA, USA), and anti-phospho-Akt (Ser473) (Cell Signaling Technology). After the incubation with primary antibody, membranes were incubated for $1 \mathrm{~h}$ at room temperature with secondary antibodies (goat anti-rabbit IgG or goat anti-mouse IgG, Jackson ImmunoResearch Laboratories, West Grove, PA, USA) diluted $1: 5,000$ in TBS-T containing $1 \%$ nonfat powdered milk. Bands were visualized by enhanced chemiluminescence (ECL) reagent (GE Healthcare Life Sciences, Piscataway, NJ, USA) and quantified by Image Studio (LI-COR, Lincoln, NE, USA). The membranes were stained with Ponceau (Sigma-Aldrich) to verify equal loading of protein across lanes.

\section{Polyubiquitinated protein concentration analysis}

The supernatants of the plantaris and soleus muscle homogenates were also used for the measurement of polyubiquitinated protein concentrations. Polyubiquitinated protein concentrations were measured with an enzyme-linked immunospecific assay (ELISA) kit according to the manufacturer's instructions (Cyclex Poly-Ubiquitinated Protein ELISA Kit; Medical \& Biological Laboratories).

\section{Serum glucose and insulin concentrations}

Serum glucose and insulin concentrations were determined with the Glucose C2 Test Wako kit (Wako Pure Chemical) and Rat Insulin ELISA Kit (Mercodia AB, Uppsala, Sweden), respectively.

\section{Muscle glycogen concentration}

For the measurement of the muscle glycogen concentration, EDL muscles were homogenized with $0.3 \mathrm{M}$ perchloric acid. The glycogen concentration was determined by the enzymatic methods of Lowry and Passonneau after acid hydrolysis (Lowry and Passonneau 1972).

\section{Statistical analysis}

All data are presented as means \pm SEM. Statistical analysis was performed by Welch's ANOVA and Bonferroni correction for post hoc analysis (Social Survey Research Information Co., Ltd., Tokyo, Japan). We performed least-squares regression analyses to examine relationships between variables. Statistical significance was defined as $\mathrm{p}<0.05$.

\section{Results}

Body weight, total intra-abdominal fat weight, and total food intake

Changes in body weights during the 2-week dietary intervention are shown in Figure 1. During the intervention period, daily calorie restriction in the Slow group for 2 weeks and 3-day fasting in the Rapid group caused a substantial reduction in body weight. The body weight in the Slow group became significantly different from the Con and Rapid groups at day $3(\mathrm{p}<0.05)$. In addition, significant body weight reduction in the Slow group from day 0 was observed at day 3 . The body weight in the Rapid group became significantly different from the Con group at day 12 (1 day after the onset of fasting). The final body weights were about $15 \%$ lower in both the Slow and Rapid groups than in the Con group $(\mathrm{p}<0.001)$ (Table 1). Total intra-abdominal fat weights were also significantly lower in both the Slow and Rapid groups than in the Con group $(p<0.001)$, with no significant differences between the Slow and Rapid groups (Table 1).

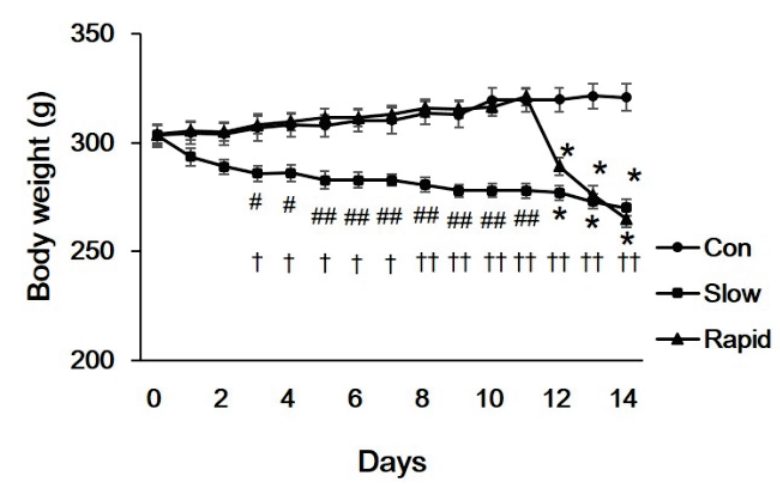

Fig. 1. Changes in the body weights of rats during a 14-day dietary intervention. Con, ad libitum-fed control group; Slow, daily calorie restriction-induced slow body weight-loss group; Rapid, fasting-induced rapid weight-loss group. Values are means \pm SEM. ${ }^{*} p<0.05$, \#\# $p<0.01$ vs. Con and Rapid, respectively; $* p<0.001$ vs. Con; ${ }^{+} p<0.05,{ }^{++} p<0.01$ vs. day 0 in Slow group, respectively. 
Table 1. Body weight, intra-abdominal fat weight, total food intake, muscle wet weight, total protein content, serum glucose, insulin and muscle glycogen concentrations in rats.

\begin{tabular}{lccc}
\hline & Con & Slow & Rapid \\
\hline Initial body weight $(g)$ & $303 \pm 5.0$ & $304 \pm 5.0$ & $304 \pm 4.0$ \\
Final body weight $(g)$ & $321 \pm 6.0$ & $270 \pm 2.0^{* * *}$ & $265 \pm 3.0^{* * *}$ \\
Intra-abdominal fat weight $(g)$ & $13.7 \pm 0.3$ & $8.5 \pm 0.5^{* * *}$ & $9.2 \pm 0.9^{* * *}$ \\
Food intake (g) & $255 \pm 7.0$ & $170 \pm 1.0^{* * *}$ & $193 \pm 3.0^{* * *}$, \\
Plantaris muscle wet weight (mg) & $287 \pm 9.0$ & $267 \pm 6.0$ & $251 \pm 6.0^{*}$ \\
Plantaris muscle protein content $(m g)$ & $56 \pm 3.0$ & $54 \pm 1.0$ & $49 \pm 1.0^{*}$ \\
Soleus muscle wet weight $(m g)$ & $114 \pm 3.0$ & $103 \pm 6.0$ & $105 \pm 2.0$ \\
Soleus muscle protein content $(m g)$ & $20 \pm 1.0$ & $18 \pm 1.0$ & $19 \pm 1.0$ \\
Serum glucose (mmol/l) & $12.4 \pm 0.5$ & $13.2 \pm 0.3$ & $11.1 \pm 0.7$ \\
Serum insulin ( $\mu$ g/l) & $7.6 \pm 0.6$ & $3.8 \pm 0.4^{* * *}$ & $1.0 \pm 0.4^{* * *}, \S$ \\
Muscle glycogen concentration $(\mu \mathrm{mol} / g$ wet tissue) & $47.1 \pm 1.2$ & $41.8 \pm 1.6$ & $27.7 \pm 1.7^{* * *,}, \S \S$ \\
\hline
\end{tabular}

Values are means $\pm \mathrm{SEM}, \mathrm{n}=5$. * and $* * *$ indicate significant differences from the values obtained in the Con group at $\mathrm{p}<0.05$ and $p<0.001$, respectively. ${ }^{\S},{ }^{\S}$ and ${ }^{\S \S}$ indicate significant differences from the values obtained in the Slow group at $p<0.05, p<0.01$ and $\mathrm{p}<0.001$, respectively.

Total food intake during the 2-week experimental period was significantly lower in the Slow and Rapid groups than in the Con group ( $\mathrm{p}<0.001$; Table 1). Furthermore, total food intake was significantly lower in the Slow group than in the Rapid group $(\mathrm{p}<0.05)$.

Serum glucose and muscle glycogen concentration

At the completion of the 14-day dietary intervention, there was no significant difference in serum glucose concentration among the three groups (Table 1). Although there was no significant difference in the glycogen concentration of EDL muscle between the Con and Slow groups, the muscle glycogen concentration was significantly lower in the Rapid group than in the Con and Slow groups $(\mathrm{p}<0.001 ;$ Table 1$)$.

\section{Muscle wet weight and muscle total protein content}

There were no significant differences in muscle wet weight and total protein content of the soleus muscle among the three groups (Table 1). Although the wet weight and total protein content of the plantaris muscle did not differ between the Con and Slow groups, the muscle weight and total protein content in the plantaris muscle were significantly lower in the Rapid group than in the Con group $(\mathrm{p}<0.05$; Table 1$)$.

\section{Autophagy-lysosome activity}

The microtubule-associated protein LC3 is now widely used to monitor the autophagy-lysosome system. The cytosolic form of LC3 (LC3-I) conjugates with phosphatidylethanolamine to form the LC3-phosphatidylethanolamine conjugate (LC3-II), which is recruited to autophagosomal membranes (Mizushima and Yoshimori 2007). Because the amount of LC3-II is correlated with the extent of autophagosome formation and an increased LC3-II/LC3-I ratio is representative of accelerated autophagy-lysosome activity (Lee et al. 2014), we determined the expression levels of LC3-I and LC3-II and used the LC3-II/LC3-I ratio as a marker of autophagy-lysosome activity.

In both plantaris and soleus muscles, LC3-II/LC3-I ratios were significantly higher in the Slow group than in the Con group ( $<<0.01$; Fig. 2 A, B). Further increases in LC3-II/LC3-I ratios were observed in the plantaris and soleus muscles of the Rapid group $(\mathrm{p}<0.001$ vs. the Con and Slow groups; Fig. 2A, B). In the plantaris muscle but not the soleus muscle, the LC3-II/LC3-I ratio was significantly and negatively associated with the muscle wet weight $(\mathrm{p}<0.01)$ and muscle protein content ( $p<0.05$; Fig. $3 \mathrm{~A}, \mathrm{C})$. 
A

Plantaris

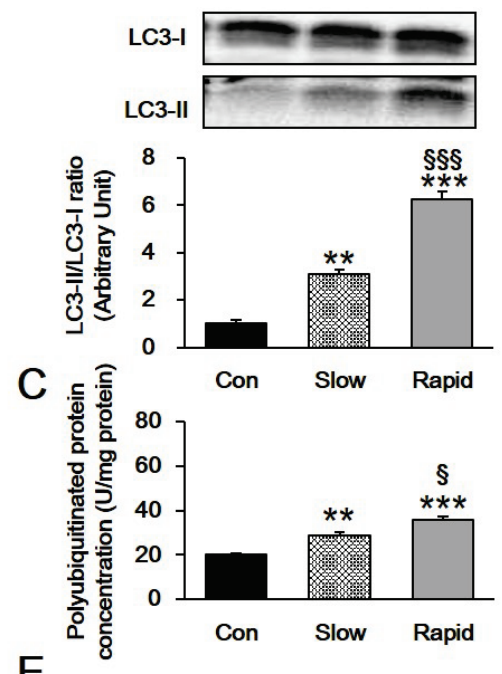

$\mathrm{E}$

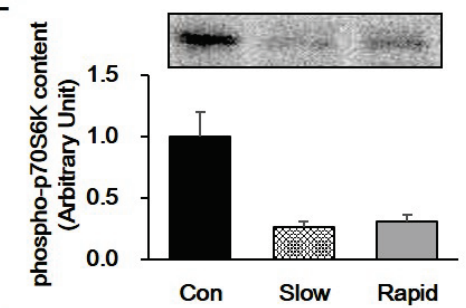

G

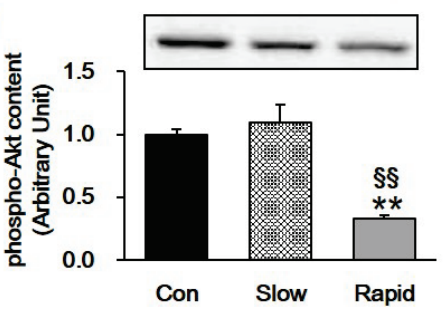

A

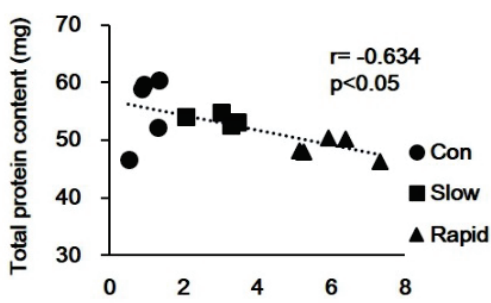

LC3-II/LC3-I ratio (Arbitrary Unit)

C

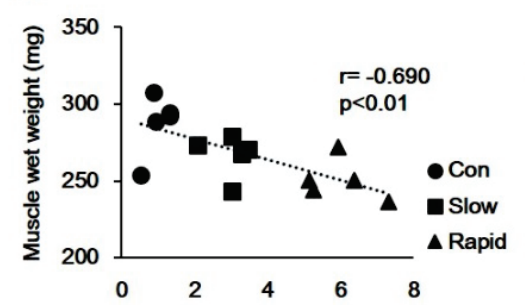

LC3-II/LC3-I ratio (Arbitrary Unit)
B

Soleus

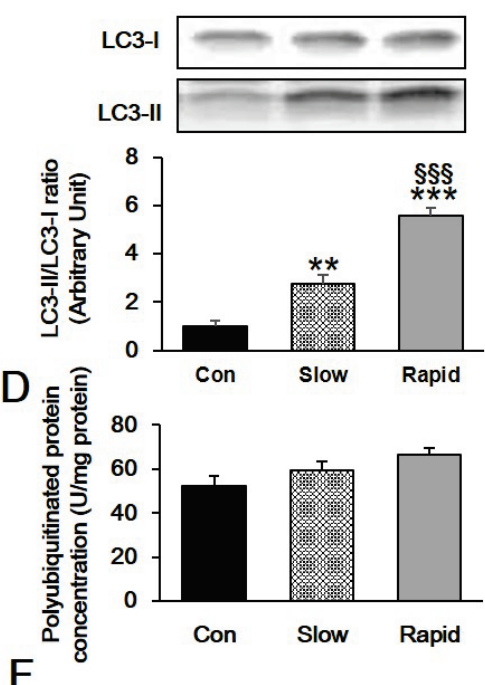

$\mathrm{F}$

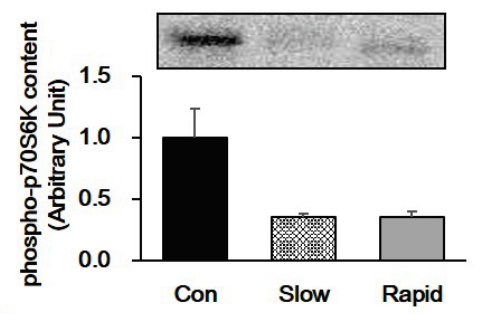

$\mathrm{H}$

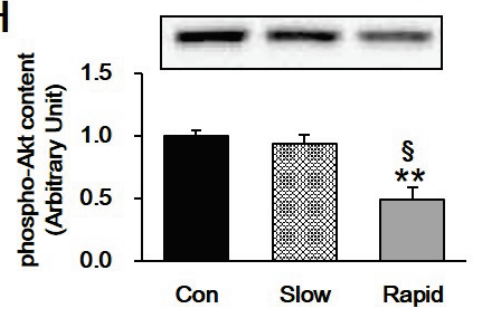

B

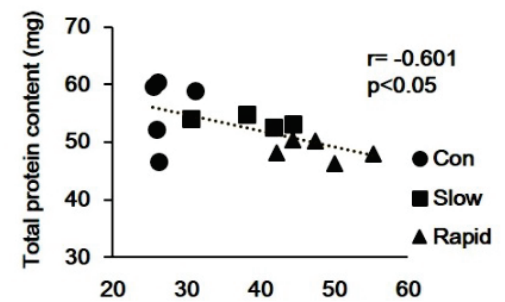

Polyubiquitinated protein concentration (U/mg protein)

D

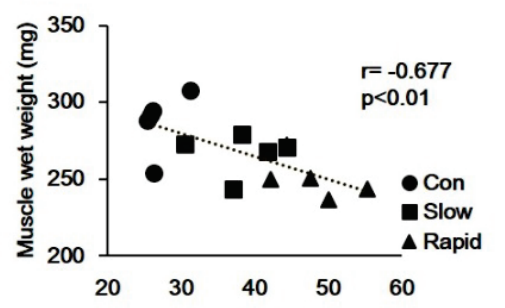

Polyubiquitinated protein concentration (U/mg protein)
Fig. 2. Effects of rapid or slow body weight loss on the LC3-II/-I ratio, polyubiquitinated protein concentration, phospho-p70S6K content, and phosphoAkt content in rat plantaris (A, C, E, and $\mathbf{G}$ ) and soleus (B, D, F, and $\mathbf{H}$ ) muscles. Con, ad libitum-fed control group; Slow, daily calorie restriction-induced slow body weight-loss group; Rapid, fasting-induced rapid weight-loss group; Values are means \pm SEM. $\quad * *$ and $\quad * * *$ indicate significant differences from the values obtained in the Con group at $p<0.01$ and $\mathrm{p}<0.001$, respectively. \$, \$\$, and $\S \S \S$ indicate significant differences from the values obtained in the Slow group at $\mathrm{p}<0.05, \quad \mathrm{p}<0.01, \quad$ and $\quad \mathrm{p}<0.001$, respectively.
Fig. 3. Correlations between the LC3-II/-I ratio (A and $\mathbf{C}$ ) and the polyubiquitinated protein concentration (B and D) and the total protein content and wet weight of rat plantaris muscle. Con, ad libitum-fed control group; Slow, daily calorie restriction-induced slow body weight-loss group; Rapid, fasting-induced rapid weight-loss group. 
Protein synthesis pathway

Although mechanistic target of rapamycin (mTOR) is a master regulator of muscle protein synthesis (Wullschleger et al. 2006), the phosphorylation status of mTOR (phospho-mTOR) does not necessarily reflect mTOR activity (Eliasson et al. 2006, Fujita et al. 2007, Miyazaki et al. 2011). Many recent studies have instead evaluated the phosphorylation of p70S6K (phospho-p70S6K), a downstream target of mTORC1, as a biomarker of mTOR activity (Jacinto and Hall 2003, Tamura et al. 2014). Although both the Slow and Rapid groups tended to have lower phospho-p70S6K content in the plantaris and soleus muscles than the Con group, the difference was not statistically significant due to a considerable variability in phospho-p70S6K levels (Fig. 2E, F).

Serum insulin concentration and phosphorylated-Akt content in skeletal muscle

The insulin-Akt axis has strong inhibitory effects on both autophagy-lysosome and ubiquitin-proteasome pathways in skeletal muscle (Price et al. 1996, Mitch et al. 1999, Lee et al. 2004, Sacheck et al. 2004, Stitt et al. 2004, Wang et al. 2006). Here, the serum insulin concentration was significantly lower in both the Slow and Rapid groups than in the Con group $(\mathrm{p}<0.001$; Table 1). In addition, the serum insulin concentration was significantly lower in the Rapid group than in the Slow group ( $<0.01$; Table 1). The levels of phospho-Akt, which is the active form of Akt, in the plantaris and soleus muscles were significantly lower in the Rapid group than in the Con and Slow groups, with no significant differences between the Con and Slow groups (Con vs. Rapid: $\mathrm{p}<0.01$; Slow vs. Rapid in plantaris muscle: $p<0.01$; Slow vs. Rapid in soleus muscle: $\mathrm{p}<0.05$; Fig. $2 \mathrm{G}, \mathrm{H})$. The serum insulin concentration significantly and negatively correlated with the LC3-II/LC3-I ratio and polyubiquitinated protein concentration in the plantaris muscle ( $p<0.01$; Fig. 4A, B). In addition, the phospho-Akt content was significantly and negatively correlated with the LC3-II/LC3-I ratio and polyubiquitinated protein concentration in the plantaris muscle $(\mathrm{p}<0.05$; Fig. 4C, D).
A
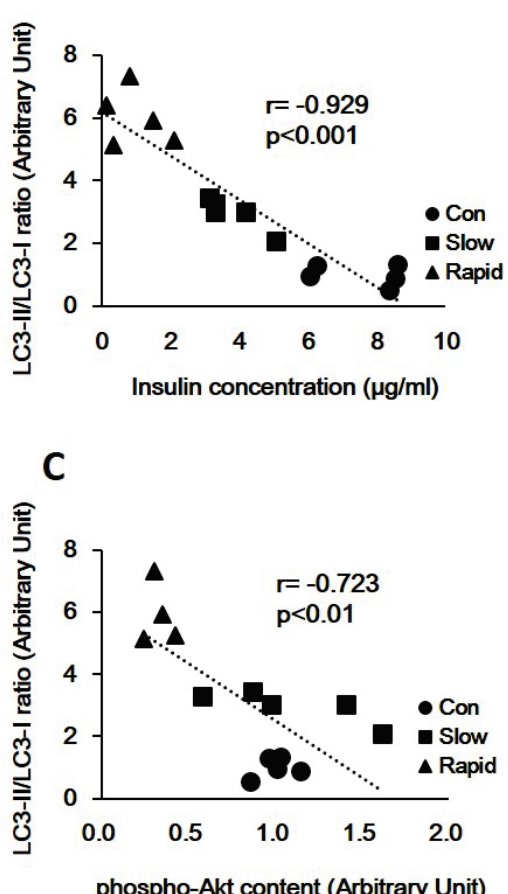

\section{Discussion}

A severe energy deficit during body weight loss causes significant reductions in skeletal muscle and body fat masses. To our knowledge, this is the first study to
Fig. 4. Correlations between the serum insulin concentration or phospho-Akt content and LC3-II/-I ratio (A and $\mathbf{C}$ ) or polyubiquitinated protein concentration (B and D) of rat plantaris muscle. Con, ad libitum-fed control group; Slow, daily calorie restriction-induced slow body weight-loss group; Rapid, fasting-induced rapid weight-loss group.

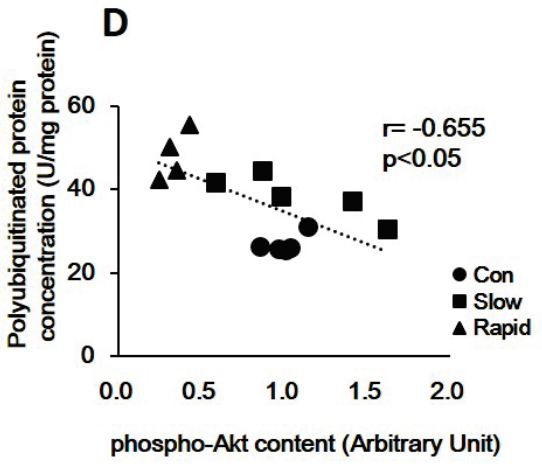

directly compare the effects of rapid and slow weight reductions, which result in acute and gradual energy deficits, respectively, on protein degradation pathways and protein content in skeletal muscle during an equivalent weight loss in rats. We found that the rapid 
weight loss induced by the 3-day fast potently activated both autophagy-lysosome and ubiquitin-proteasome pathways (Fig. 2). This fast resulted in significant reductions in the total protein content and wet weight of the fast-twitch plantaris muscle (Table 1), although both weight-loss methods decreased rat body weight and total intra-abdominal fat mass to a similar extent (Table 1 and Fig. 1).

Muscle atrophy occurs when protein degradation rates exceed protein synthesis rates. Although the phospho-p70S6K contents of the plantaris and soleus muscles appeared to be lower in both the Slow and Rapid groups than in the Con group, the difference was not statistically significant (Fig. 2E, F). In addition, the phospho-p70S6K contents of the plantaris muscle were almost identical in both weight-loss groups. It is therefore unlikely that fasting-induced atrophy in the plantaris muscle of the Rapid group was due to a diminished protein synthesis rate, although we did not directly evaluate the muscle protein synthesis rate.

Even though the total food intake during the 14-day dietary intervention was significantly higher in the Rapid group than in the Slow group, the muscle glycogen concentration was substantially lower in the Rapid group, suggesting that only the 3-day fast resulted in a severe energy deficit in muscle cells. A severe energy deficit induced by fasting and starvation activates autophagymediated protein degradation (Mizushima et al. 2004). In this study, we found that the LC3-II/LC3-I ratio, frequently used as a biomarker of autophagy-lysosome activity (Lee et al. 2014), was significantly higher in the plantaris muscle of the Rapid group than in that of both the Slow and Con groups (Fig. 2). This finding indicates that the autophagy-lysosome pathway was more potently activated in the Rapid group than in the Slow group, despite the equivalent weight loss. In addition, the LC3-II/LC3-I ratio was significantly and negatively correlated with the total protein content and muscle wet weight in the plantaris muscle (Fig. 3A, C). Based on these results, it is plausible that the higher autophagylysosome activity was responsible for the rapid weight loss-induced atrophy in the plantaris muscle.

Our results also indicate that another protein degradation pathway, the ubiquitin-proteasome system, might be involved in the muscular atrophy in the Rapid group. In the ubiquitin-proteasome system, proteins are targeted for degradation by the $26 \mathrm{~S}$ proteasome through covalent attachment of a chain of ubiquitin molecules (Goldberg 2003). We determined the polyubiquitinated protein concentration in skeletal muscle and found that the plantaris muscle in the Rapid group had a significantly higher polyubiquitinated protein concentration (Fig. 2C), as well as LC3-II/LC3-I ratio, than the Con and Slow groups. Similar to the LC3-II/LC3-I ratio, the polyubiquitinated protein concentration was significantly and negatively associated with total protein content and muscle wet weight in the plantaris muscle (Fig. 3D). These results provide strong evidence that the two major proteolytic pathways are more strongly activated during rapid body weight loss than during slow body weight reduction, resulting in a significant loss of total protein content and wet weight of fast-twitch muscle.

Insulin and its downstream effector Akt strongly inhibit both autophagy-lysosome and ubiquitinproteasome pathways in skeletal muscle (Price et al. 1996, Mitch et al.1999, Lee et al. 2004, Sacheck et al. 2004, Stitt et al. 2004, Wang et al. 2006). Whereas the serum insulin concentration and phospho-Akt content of the plantaris muscle were almost negligible in the Rapid group, the Slow group rats maintained a serum insulin concentration and phospho-Akt content similar to that of the ad libitum-fed Con group rats (Table 1 and Fig. 2G). In addition, the serum insulin concentration and phosphoAkt content were negatively correlated with the LC3-II/LC3-I ratio and polyubiquitinated protein concentration in the plantaris muscle (Fig. 4). Taken together, it is likely that the slow body weight reduction induced by daily calorie restriction can partially prevent muscular atrophy during weight loss, at least in part by maintaining the serum insulin level and its signaling pathway in skeletal muscle. Because it has been well documented that blood insulin level substantially decreases even after overnight fasting, we could not rule out the possibility that the higher proteolytic activities observed in the muscles of the Rapid group might be reflecting an acute effect of fasting (acute insulin deficiency) rather than chronic effects. However, Ogata et al. (2010) reported that LC3-II content in rat skeletal muscle did not increase in response to 1-day fasting, whereas 3-day fasting induced significant and large increase in muscle LC3-II content. It is therefore plausible that higher proteolytic activities in the Rapid group resulted from chronic and accumulated effects of 3-day fasting, but not acute effect. Future studies are required to measure the proteolytic activities in the Con and Slow group in the fasting condition, or in the Rapid group after a few hours feeding in order to assess the 
chronic adaptations and differentiate them from potential acute effects.

As shown in Table 1, the soleus muscle, unlike the plantaris muscle, did not show any atrophic changes in response to either the rapid or slow body weight reduction. Our results support a previous finding that the degree of fasting-induced atrophy is greater in fast-twitch muscle than slow-twitch muscle (Li and Goldberg 1976, Frayn and Maycock 1979). Ogata et al. (2010) reported that a fasting-induced increase in LC3-II expression was notably greater in rat fast-twitch plantaris muscle than in slow-twitch soleus muscle. Consistent with these results, we observed that the magnitudes of the increase in the LC3-II/LC3-I ratio after rapid and slow body weight reductions appeared to be relatively lower in soleus than plantaris muscle (Fig. 2A, B), providing further evidence that the autophagy pathway is preferentially induced in fast-twitch muscle in an energy deficient state. Another major finding of the present study was that the polyubiquitinated protein concentration was markedly higher in the soleus muscle than in the plantaris muscle under basal conditions (the Con group) and that it did not increase in response to fasting and daily calorie restriction (Fig. 2C, D). The blunted responses in the ubiquitin-proteasome and autophagy-lysosome systems might be associated with the atrophy resistance of soleus muscle to a severe energy deficit.

This study has several limitations. First, muscle strength and exercise capacity after the rapid or slow weight loss were not assessed in this study. Thus, we could not clarify which weight loss strategy is effective in improving exercise performance although slow weight loss induced by calorie restriction could maintain muscle mass. Second, we did not evaluate the effects of fasting or calorie restriction in combination with exercise on muscle protein content and muscle weight. The results obtained in this study cannot be directly extrapolated to athletic population, who engage in exercise training. Future extensive studies are required to examine the combined effects of exercise and dietary interventions on muscle functions as well as muscle mass in order to elucidate whether exercise training can prevent muscle atrophy induced by weight loss.

\section{Conclusions}

During an equivalent weight loss, the rapid weight loss induced by short-term fasting more strongly activates autophagy-lysosome and ubiquitin-proteasome pathways than a slow body weight reduction induced by daily calorie restriction, resulting in muscular atrophy in fast-twitch plantaris muscle but not in soleus muscle.

\section{Conflict of Interest}

There is no conflict of interest.

\section{Acknowledgements}

This work was supported by JSPS KAKENHI Grant Numbers JP15K01615, JP25750330, and JP16J10555 (to S. Terada).

\section{References}

BUJAK AL, CRANE JD, LALLY JS, FORD RJ, KANG SJ, REBALKA IA, GREEN AE, KEMP BE, HAWKE TJ, SCHERTZER JD, STEINBERG GR: AMPK activation of muscle autophagy prevents fasting-induced hypoglycemia and myopathy during aging. Cell Metab 21: 883-890, 2015.

CHOMA CW, SFORZO GA, KELLER BA: Impact of rapid weight loss on cognitive function in collegiate wrestlers. Med Sci Sports Exerc 30: 746-749, 1998.

ELIASSON J, ELFEGOUN T, NILSSON J, KÖHNKE R, EKBLOM B, BLOMSTRAND E: Maximal lengthening contractions increase p70 S6 kinase phosphorylation in human skeletal muscle in the absence of nutritional supply. Am J Physiol Endocrinol Metab 291: E1197-E1205, 2006.

FRAYN KN, MAYCOCK PF: Regulation of protein metabolism by a physiological concentration of insulin in mouse soleus and extensor digitorum longus muscles. Effects of starvation and scald injury. Biochem J 184: 323-330, 1979.

FUJITA S, ABE T, DRUMMOND MJ, CADENAS JG, DREYER HC, SATO Y, VOLPI E, RASMUSSEN BB: Blood flow restriction during low-intensity resistance exercise increases $\mathrm{S} 6 \mathrm{~K} 1$ phosphorylation and muscle protein synthesis. J Appl Physiol 103: 903-910, 2007.

GOLDBERG AL: Protein degradation and protection against misfolded or damaged proteins. Nature 426: 895-899, 2003. 
JACINTO E, HALL MN: TOR signalling in bugs, brain and brawn. Nat Rev Mol Cell Biol 4: 117-126, 2003.

JAGOE RT, LECKER SH, GOMES M, GOLDBERG AL: Patterns of gene expression in atrophying skeletal muscles: response to food deprivation. FASEB $J$ 16: 1697-1712, 2002.

LEE JH, LEE JH, JIN M, HAN SD, CHON GR, KIM IH, KIM S, KIM SY, CHOI SB, NOH YH: Diet control to achieve euglycemia induces significant loss of heart and liver weight via increased autophagy compared with ad libitum diet in diabetic rats. Exp Mol Med 46: e111, 2014.

LEE SW, DAI G, HU Z, WANG X, DU J, MITCH WE: Regulation of muscle protein degradation: coordinated control of apoptotic and ubiquitin-proteasome systems by phosphatidylinositol 3 kinase. J Am Soc Nephrol 15: 1537-1545, 2004.

LI JB, GOLDBERG AL: Effects of food deprivation on protein synthesis and degradation in rat skeletal muscles. $\mathrm{Am}$ J Physiol 231: 441-448, 1976.

LOWRY OH, PASSONNEAU JV: A Flexible System of Enzymatic Analysis. Academic Press, New York, 1972, p 291.

MITCH WE, BAILEY JL, WANG X, JURKOVITZ C, NEWBY D, PRICE SR: Evaluation of signals activating ubiquitin-proteasome proteolysis in a model of muscle wasting. Am J Physiol 276: C1132-C1138, 1999.

MITCH WE, GOLDBERG AL: Mechanisms of muscle wasting. The role of the ubiquitin-proteasome pathway. $N$ Engl J Med 335: 1897-1905, 1996.

MIYAZAKI M, MCCARTHY JJ, FEDELE MJ, ESSER KA: Early activation of mTORC1 signalling in response to mechanical overload is independent of phosphoinositide 3-kinase/Akt signalling. $J$ Physiol 589: 1831-1846, 2011.

MIZUSHIMA N, YAMAMOTO A, MATSUI M, YOSHIMORI T, OHSUMI Y: In vivo analysis of autophagy in response to nutrient starvation using transgenic mice expressing a fluorescent autophagosome marker. Mol Biol Cell 15: 1101-1111, 2004.

MIZUSHIMA N, YOSHIMORI T: How to interpret LC3 immunoblotting. Autophagy 3: 542-545, 2007.

MORTIMORE GE, PÖSÖ AR: Intracellular protein catabolism and its control during nutrient deprivation and supply. Annu Rev Nutr 7: 539-564, 1987.

OGATA T, OISHI Y, HIGUCHI M, MURAOKA I: Fasting-related autophagic response in slow- and fast-twitch skeletal muscle. Biochem Biophys Res Commun 394: 136-140, 2010.

PRICE SR, BAILEY JL, WANG X, JURKOVITZ C, ENGLAND BK, DING X, PHILLIPS LS, MITCH WE: Muscle wasting in insulinopenic rats results from activation of the ATP-dependent, ubiquitin-proteasome proteolytic pathway by a mechanism including gene transcription. J Clin Invest 98: 1703-1708, 1996.

RELJIC D, HÄSSLER E, JOST J, FRIEDMANN-BETTE B: Rapid weight loss and the body fluid balance and hemoglobin mass of elite amateur boxers. $J$ Athl Train 48: 109-117, 2013.

ROCK KL, GRAMM C, ROTHSTEIN L, CLARK K, STEIN R, DICK L, HWANG D, GOLDBERG AL: Inhibitors of the proteasome block the degradation of most cell proteins and the generation of peptides presented on MHC class I molecules. Cell 78: 761-771, 1994.

SACHECK JM, OHTSUKA A, MCLARY SC, GOLDBERG AL: IGF-I stimulates muscle growth by suppressing protein breakdown and expression of atrophy-related ubiquitin ligases, atrogin-1 and MuRF1. Am J Physiol Endocrinol Metab 287: E591-E601, 2004.

STITT TN, DRUJAN D, CLARKE BA, PANARO F, TIMOFEYVA Y, KLINE WO, GONZALEZ M, YANCOPOULOS GD, GLASS DJ: The IGF-1/PI3K/Akt pathway prevents expression of muscle atrophyinduced ubiquitin ligases by inhibiting FOXO transcription factors. Mol Cell 14: 395-403, 2004.

TAMURA Y, MATSUNAGA Y, MASUDA H, TAKAHASHI Y, TAKAHASHI Y, TERADA S, HOSHINO D, HATTA H: Postexercise whole body heat stress additively enhances endurance training-induced mitochondrial adaptations in mouse skeletal muscle. Am J Physiol Regul Integr Comp Physiol 307: R931-R943, 2014.

WANG X, HU Z, HU J, DU J, MITCH WE: Insulin resistance accelerates muscle protein degradation: Activation of the ubiquitin-proteasome pathway by defects in muscle cell signaling. Endocrinology 147: 4160-4168, 2006.

WULLSCHLEGER S, LOEWITH R, HALL MN: TOR signaling in growth and metabolism. Cell 124: 471-484, 2006. 